\title{
SFCOMPO 2.0 - A relational database of spent fuel isotopic measurements, reactor operational histories, and design data
}

\author{
Franco Michel-Sendis ${ }^{1, \text { a }}$, Jesus Martinez-González ${ }^{2}$, and Ian Gauld ${ }^{3}$ \\ 1 OECD Nuclear Energy Agency, France \\ 2 Universidad Politecnica de Madrid, Spain \\ 3 Oak Ridge Nuclear Laboratory, USA
}

\begin{abstract}
SFCOMPO-2.0 is a database of experimental isotopic concentrations measured in destructive radiochemical analysis of spent nuclear fuel (SNF) samples. The database includes corresponding design description of the fuel rods and assemblies, relevant operating conditions and characteristics of the host reactors necessary for modelling and simulation. Aimed at establishing a thorough, reliable, and publicly available resource for code and data validation of safety-related applications, SFCOMPO-2.0 is developed and maintained by the OECD Nuclear Energy Agency (NEA). The SFCOMPO-2.0 database is a Java application which is downloadable from the NEA website.
\end{abstract}

\section{Introduction}

The technical capability to accurately predict spent fuel isotopic compositions is one of the foundations upon which all safety-related applications and licensing evaluations involving spent nuclear fuel (SNF) are based. A sound experimental database of measured isotopic concentrations, with uncertainties, design specifications and operational history data is therefore required for the proper validation of depletion methodologies (codes and data) which are applied to safety-related studies. Having a centralised and internationally reviewed database of SNF assay data for a large selection of international reactor designs is of obvious interest to the nuclear energy scientific community in general.

The NEA and ORNL have collaborated in recent years on a major development effort which has resulted in the production of the SFCOMPO-2.0 database, which is briefly presented in this paper.

\section{Background}

\subsection{From SFCOMPO to SFCOMPO-2.0}

In the early 1990's, in view of building an experimental database for validation of codes used in burn-up calculations, the Japan Atomic Energy Research Institute (JAERI) initiated the compilation of existing nuclide composition data of SNF obtained from the open literature of post-irradiation experiments. In subsequent years this led to the creation of the first version of the spent nuclear fuel database named SFCOMPO, which consisted of a series of searchable HTML Web pages which contained data coming from 246 samples taken from 7 PWR reactors and 7 BWR reactors. In 2001, this version of SFCOMPO was migrated to the NEA web server, and had little development until SFCOMPO-2.0. The

a e-mail: franco.michelsendis@oecd.org original SFCOMPO was discontinued in 2016, replaced by SFCOMPO-2.0.

The SFCOMPO-2.0 relational database presented in this paper is a downloadable JAVA application (www. oecd-nea.org/sf compo/) that contains data from 44 different reactors, representing 8 international reactor designs (AGR, BWR, CANDU, MAGNOX, PWR, RBMK, VVER-1000, VVER-440), giving access to over 750 measured SNF samples.

SFCOMPO-2.0 aims at providing access to open experimental assay data of SNF in order to ensure their preservation and facilitate their qualification as evaluated assay data suitable for the validation of methodologies used to predict the composition of irradiated nuclear fuel.

Like other existing NEA databases and visualization tools, SFCOMPO-2.0 helps the analyst or user in identifying data that is best suited for their specific application and facilitates access to the primary sources of relevant reference information.

\section{Assay data}

Assay data are sets of measured nuclide concentrations of well-characterised spent nuclear fuel samples, complemented with reactor operational data describing the irradiation of the samples, in sufficient detail for it to be potentially used as a depletion benchmark model. The review and evaluation of assay data is therefore a multidisciplinary task involving experts in radiochemistry, reactor operation, spent fuel modeling, and simulation, among others. These different expertise domains are represented within the NEA Expert Group on Assay Data of Spent Nuclear Fuel (EGADSNF) [1], which is responsible for the overview of SFCOMPO development and has proposed clear guidelines for the evaluation of assay data before they are considered fully suitable for code benchmarking uses (for a given application) [2,3]. 


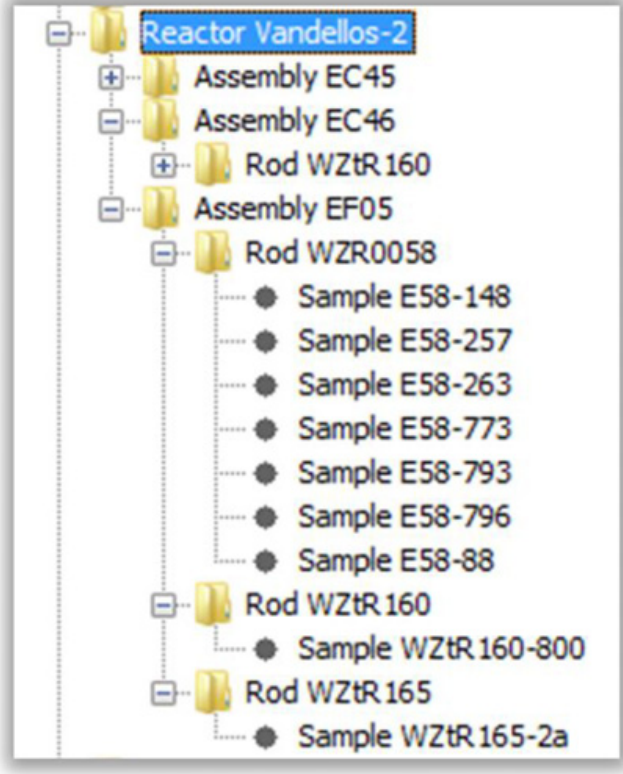

Figure 1. Screen capture of the browser window in SFCOMPO-2.0 showing the different hierarchy levels of the database.

An assay data set for validation of depletion calculations therefore contains a broad variety of information such as:

- Radiochemical data, measured compositions or activities.

- Measured nuclide compositions of the sample.

- Experimental derivation of an estimated burn-up of the sample.

- Relevant geometry of reactor / assembly / rod, sample location, reactor design data.

- Location of the sample within the reactor.

- Immediate vicinity configuration.

- Operational History.

- Irradiation history and cooling times, down times.

- Power history, temperature histories, concentration of neutron poisons, all other time-depending parameters as needed or as available.

- Original lab reports documenting the data.

SFCOMPO-2.0 organizes and gives acess to these different varieties of information in an user-friendly manner. The database stores all data in a hierarchical vertical tree structure which has 5 main levels of description. These 5 levels are, in descending order, Reactor, Assembly, Rod, Sample and Measurements. Figure 1 illustrates this hierarchy as would be seen by an user browsing the database. Each level has additional horizontal attributes which describe that level and these entries are mandatory. The temporal evolution (operational history data) of any physical parameter can be stored in the database and bound to the appropriate level. Figures 2 and 3 are views of the graphical user interface of SFCOMPO-2.0.

\section{SFCOMPO-2.0 features}

\subsection{Access to SFCOMPO-2.0}

SFCOMPO-2.0 can be downloaded from the internet free of charge, and is currently accessed through a password
Table 1. Reactor entries contained in SFCOMPO-2.0.

\begin{tabular}{|c|c|}
\hline Reactor Type & Reactor Unit / Name \\
\hline \multirow{3}{*}{ AGR } & Hinkley-3 \\
\hline & Hinkley-4 \\
\hline & Hunterston B-1 \\
\hline \multirow{12}{*}{ BWR } & Cooper-1 \\
\hline & Dodewaard-1 \\
\hline & Forsmark-3 \\
\hline & Fukushima-Daiichi-3 \\
\hline & Fukushima-Daini-1 \\
\hline & Fukushima-Daini-2 \\
\hline & Garigliano-1 \\
\hline & Gundremmingen-1 \\
\hline & JPDR-1 \\
\hline & Monticello-1 \\
\hline & Quad Cities-1 \\
\hline & Tsuruga-1 \\
\hline \multirow{3}{*}{ CANDU } & Bruce-1 \\
\hline & NPDR-1 \\
\hline & Pickering A-1 \\
\hline \multirow{2}{*}{ MAGNOX } & Bradwell-1 \\
\hline & Hunterston A-1 \\
\hline \multirow{16}{*}{ PWR } & Beznau-1 \\
\hline & Calvert-Cliffs-1 \\
\hline & Genkai-1 \\
\hline & Gösgen-1 \\
\hline & H.B.Robinson-2 \\
\hline & Mihama-3 \\
\hline & Neckarwestheim-2 \\
\hline & Obrigheim-1 \\
\hline & Ohi-1 \\
\hline & Ohi-2 \\
\hline & Takahama-3 \\
\hline & Three Mile Island-1 \\
\hline & Trino Vercellese-1 \\
\hline & Turkey Point-3 \\
\hline & Vandellos-2 \\
\hline & Yankee-1 \\
\hline RBMK & Leningrad-1 \\
\hline \multirow{4}{*}{ VVER-1000 } & Balakovo-2 \\
\hline & Balakovo-3 \\
\hline & Kalinin-1 \\
\hline & Novovoronezh-5 \\
\hline \multirow{3}{*}{ VVER-440 } & Kola-3 \\
\hline & Novovoronezh-3 \\
\hline & Novovoronezh-4 \\
\hline
\end{tabular}

which can be obtained from the NEA, if you are an NEA member country national. Requests for access can be sent to sfcompo@oecd-nea.org. Access is granted on an individual basis and the user must have or create an NEA account which will provide him/her with a username and a password.

\subsection{Data in SFCOMPO-2.0}

The philosophy behind SFCOMPO-2.0 is to preserve the original information found in the primary reference reports. For this reason, all measured data is captured as published in the original units provided by the primary sources.

The database contains more than 20000 measurement points representing over 750 samples.

The data in SFCOMPO-2.0 have been independently reviewed for consistency with the experimental 


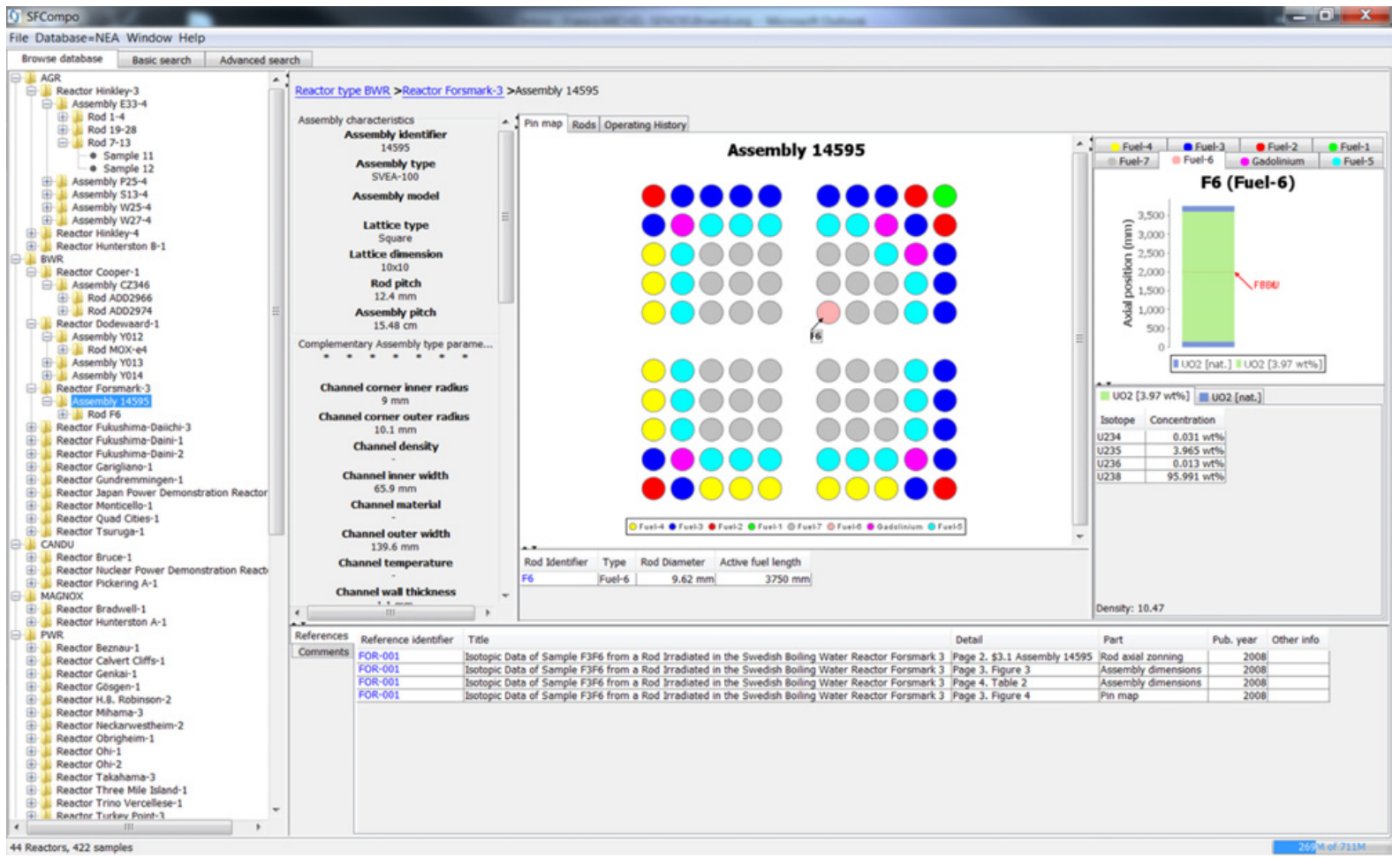

Figure 2. A view of the SFCOMPO-2.0 graphical interface displaying the geometry of the selected case, here the BWR Forsmark-3 assembly, with information on design details, sample location, initial composition and links to the original documents.

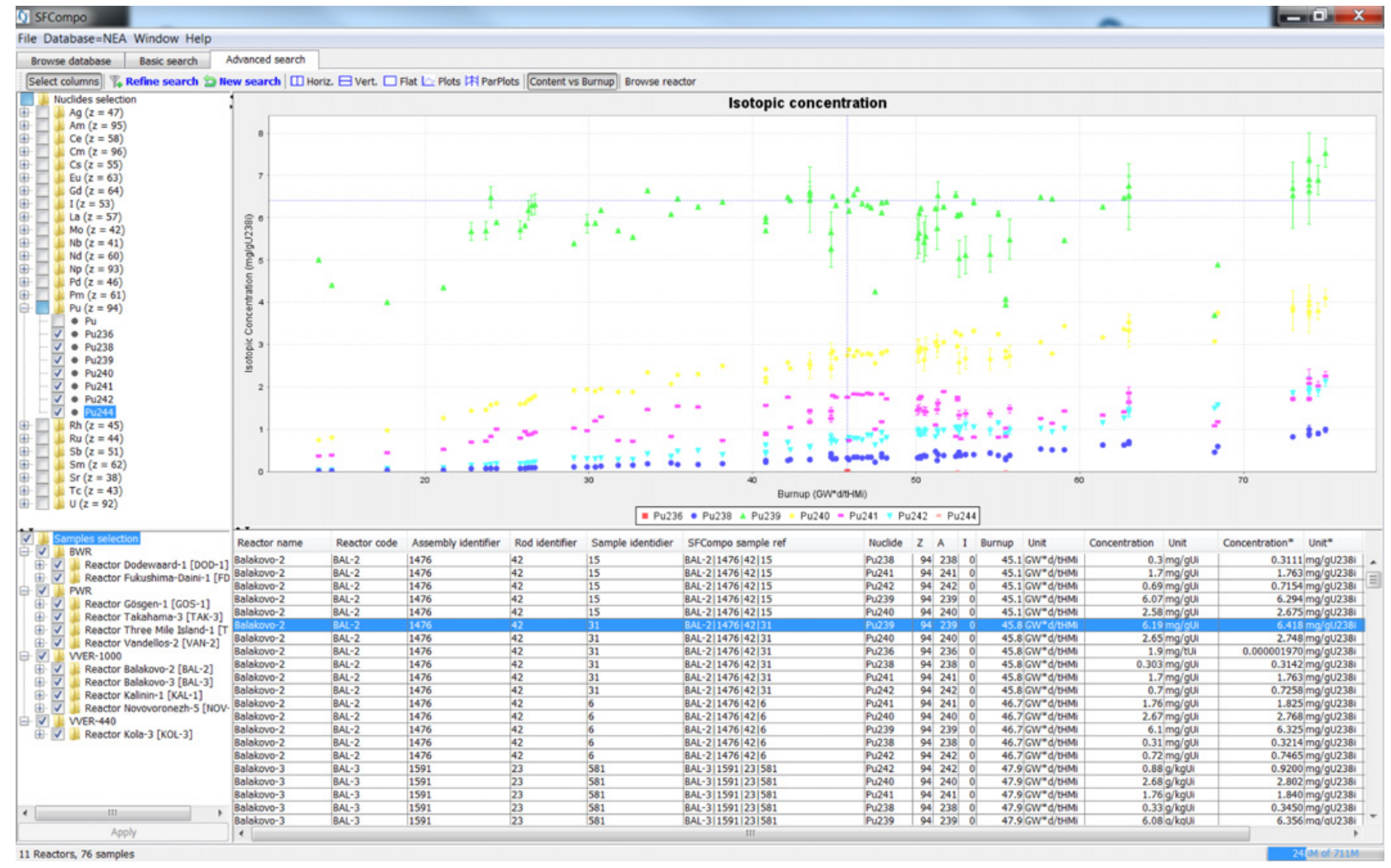

Figure 3. A view of the SFCOMPO-2.0 graphical interface displaying results of a search, here Pu isotopics for a different selection of reactor types. A graphic plotter allows for visualization of trends, while a tabular panel provides numeric data. Clicking on a point in the graph will reveal the numerical data entry in the tabular panel. Plots and tables can easily be exported by the user. 
reports. A comprehensive review was extensively performed by one of the authors (Martinez-Gonzalez) to ensure standardization of all SFCOMPO entries. While the data in SFCOMPO-2.0 have been independently reviewed and cross-checked for consistency with the primary sources, these data have not been formally evaluated.

Any errors in measurements, omissions, or inconsistencies in the original reported data may therefore be reproduced in the database. It is therefore important that any user of the data for code validation consider and assess the potential data deficiencies. The evaluation of assay data will provide a more complete assessment and may result in the development of benchmark specifications in cases of high quality experiments..

\subsection{Search criteria}

The application implements two search panels (Basic and Advanced) which allow to filter the data according to the following critera:

- Reactor type, reactor name.

- Sample Fuel type.

- Estimated Burnup (range).

- Measured Nuclide.

- Coolant, Moderator types.

- Assembly Identifier.

- Lattice types, Lattice dimensions.

- Fuel Rod pitch, Fuel Rod diameter.

- Enrichment range.

- Axial position range.

- Measurement Type.

- Measurement Uncertainty (range).

\subsection{References and comments in SFCOMPO-2.0}

SFCOMPO-2.0 allows for a unique reference to be assigned to each sample and equally to clearly reference all data to their primary source. Comments at every level of the database may also be entered. Primary sources such as laboratory reports and evaluator reports can be accessed through an in-built PDF viewer if the reference reports (which can be provided separately through the SFCOMPO website) are locally installed.

\subsection{Importing proprietary data into SFCOMPO}

A user wishing to enter proprietary data into a local installation of the database may do so by previously filling and importing a spreadsheet template (which can be provided by NEA upon request), with an import function designed for this use.

\section{Conclusions}

The Expert Group on Assay Data of Spent Nuclear Fuel provides an international framework for compilation and technical review of available assay data for benchmarking purposes. Since 2012, the NEA Data Bank undertook a significant development effort that led to the development of the new database of Spent Nuclear Fuel nuclide composition SFCOMPO-2.0. In strong collaboration with Oak Ridge National Laboratory [4,5], the effort to compile new reactor datasets expanded the representation of reactors in the original SFCOMPO database to an international selection of designs and a modern, functional database with many functionalities. More information can be obtained at www. oecd-nea.org/sf compo.

\section{References}

[1] WPNCS Expert Group on Assay Data of Spent Nuclear Fuel (ADNSF), http://www.oecdnea.org/science/wpncs/ADSNF

[2] OECD NEA - Evaluation Guide for the Evaluated Spent Nuclear Fuel Assay Database SFCOMPO, available online at https://www.oecd-nea.org/ science/docs/2015/nsc-r2015-8.pdf

[3] OECD NEA - Spent Nuclear Fuel Assay Data for Isotopic Validation, State-of-the-art Report, available online at http://www.oecd-nea.org/science/ wpncs/ADSNF/SOAR_final.pdf (2011)

[4] F. Michel-Sendis et al, "A New OECD NEA Database of nuclide compositions of Spent Nuclear Fuel", Proceedings of the International Conference PHYSOR 2014, Kyoto, Japan

[5] I. Gauld et al., "OECD NEA Benchmark Database of Spent Nuclear Fuel Isotopic Compositions for World Reactor Designs", Proceedings of the International Conference PHYSOR 2014, Kyoto, Japan 